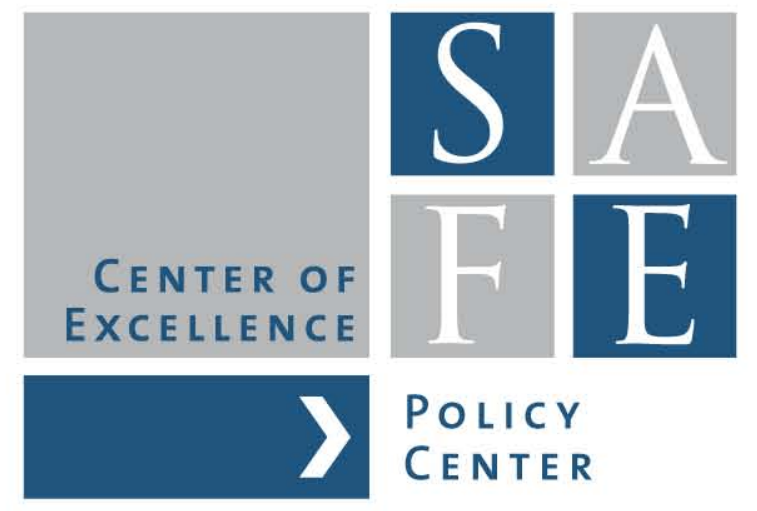

Jan Pieter Krahnen

\title{
Rettung durch Regulierung? Eckpunkte des Liikanen-Berichts
}

White Paper Series No. 8

Center of Excellence SAFE Sustainable Architecture for Finance in Europe A cooperation of the Center for Financial Studies and Goethe University Frankfurt 
Dieses Papier wurde für das Symposium des Vereins für Socialpolitik zum Thema "Europäische Bankenunion: Ein neuer Ordnungsrahmen?", am 16. September 2013 in Frankfurt am Main, angefertigt und wird in der Zeitschrift "Perspektiven der Wirtschaftspolitik" erscheinen. 


\title{
Rettung durch Regulierung? Eckpunkte des Liikanen-Berichts
}

\author{
Jan Pieter Krahnen i, ii
}

\section{Zusammenfassung}

Ausgehend von einer Erläuterung der Kriseninterpretation (crisis narrative), wie sie in dem Bericht der Liikanen-Kommission zugrunde liegt, werden die nach Ansicht des Verfassers zentralen Vorschläge des Kommissionsberichts ausgewählt, vorgestellt und in den größeren Rahmen einer erneuerten Ordnungspolitik für die Finanzmärkte Europas eingeordnet. Die mit den Vorschlägen eng zusammenhängenden Reformelemente der Bankenunion werden in diesem Text bewusst ausgeklammert. Die beiden zentralen Strukturvorschläge des Liikanen-Berichts betreffen die Abspaltung der Handelsgeschäfte von dem Universalbankengeschäft für große, internationale Banken (der Trennbankenvorschlag), sowie die verpflichtende Emission nachrangigen, glaubwürdig haftenden Fremdkapitals (der strenge Bail-in Vorschlag). Glaubwürdigkeit der Haftungszusage wird durch strenge Halterestriktionen erreicht. Vorhersehbare Folgerungen einer Einführung dieser Strukturregeln für die Finanzindustrie und -märkte werden in einem abschließenden Teil angesprochen.

i. Fachbereich Wirtschaftswissenschaften, House of Finance, Goethe-Universität Frankfurt [www.finance.unifrankfurt.de], CFS und Center of Excellence-SAFE [www.safe-frankfurt.de], Email: krahnen@finance.unifrankfurt.de, Tel.: +49-69-798-33699.

ii. Der Verfasser war Mitglied der High-level Expert Group on reforming the structure of the EU banking sector (Liikanen Kommission 2012). Für die in diesem Aufsatz vorgenommene Auswahl an Themen und für deren weitergehende Interpretation ist allein der Autor verantwortlich; sie stellt keine Stellungnahme der HLEG dar. Für hilfreiche Kommentare während des Symposiums des Vereins für Socialpolitik zum Thema "Europäische Bankenunion: Ein neuer Ordnungsrahmen?" am 16. September 2013 in Frankfurt am Main danke ich Horst Gischer, Frank Heinemann, Thomas Mayer und Hermann Remsperger. 


\section{Rettung durch Regulierung? Eckpunkte des Liikanen-Berichts}

\section{Gliederung}

1. Einleitung

2. Der Liikanen-Bericht: Deutung der Krise und wichtige Reformvorschläge
a. Die Krisenerklärung
b. Trennung der Handelsaktivitäten vom Universalbankengeschäft: Abwicklung erleichtern
c. Nachrangbankanleihen für Nichtbanken: Ansteckung vermindern

3. Folgerungen für Banken, Investoren und Aufsicht

a. Folgeüberlegungen zum Trennbanken-Vorschlag

i. Verminderte Profitabilität?

ii. Aufsichtsarbitrage?

iii. Weniger Liquidität?

b. Folgeüberlegungen zur Schaffung von Bail-in Kapital

i. Wer ist Risikoträger für Bail-in Fremdkapital?

ii. Entsteht ein ausreichend großer Markt?

iii. Welche Anforderungen stellen sich an die Aufsicht?

4. Ausblick und offene Fragen

5. Quellenverzeichnis 


\section{Einleitung}

Von den Organisatoren dieser Tagung kommt der Vorschlag, vorliegende Konzepte zu einer Europäischen Bankenunion aus ökonomischer (und politischer) Sicht zu prüfen. Die auf den folgenden Seiten dargelegte Grundargumentation der Vorschläge des Liikanen-Berichts stellt ungeachtet meiner Zugehörigkeit zu dem Autorenteam eine persönliche Interpretation der Hauptempfehlungen des Berichts dar. Dabei enthält die Vorsilbe (,Haupt-') bereits eine wertende Auswahl, wie sie aus der Lektüre des Berichts nicht unmittelbar ersichtlich ist. Diese Wertung bezieht sich auf die Bedeutungsschwere einzelner Empfehlungen für die beabsichtigte Wiederherstellung marktwirtschaftlicher Verhältnisse im Bankensektor. ${ }^{1}$

Der Liikanen-Bericht und die darin enthaltenen Vorschläge für Strukturreformen im Bankensektor sind vor knapp einem Jahr (genauer Anfang Oktober 2012) veröffentlicht worden. Mit einer offiziellen Antwort der Europäischen Kommission als Adressatin des Berichts wird gegen Jahresende 2013 gerechnet. Die Tagung findet daher zu einem Zeitpunkt statt, der einen abwägenden Blick auf diese Vorschläge im Kontext der Bankenunion als Gesamtpaket gestattet.

Verschiedene Aspekte der sich abzeichnenden umfassenden Bankenunion (BU) befinden sich noch in der Schwebe. Von den vier Säulen der BU - einheitliches Restrukturierungs- und Abwicklungsregime (Recovery and Resolution Directive, RRD), Vergemeinschaftung der mikro-prudentiellen Bankenaufsicht (Single Supervisory Mechansim, SSM), gemeinsame Abwicklungsbehörde (Single Resolution Agency, SRA) und gemeinschaftliche Einlagensicherung (Deposit Guarantee Scheme, DGS) - sind lediglich die ersten beiden klar umrissen und befinden sich im Prozess der Endabstimmung mit dem Europäischen Parlament (RRD) bzw. der Implementierung (SSM). In beiden Fällen ist die rechtliche Belastbarkeit einer Kompetenzverlagerung von nationalen auf europäische Institutionen nach wie vor strittig. ${ }^{2}$

Ein Entwurf für eine Europäische Abwicklungsbehörde (SRA) ist kürzlich von der Kommission vorgelegt worden. Dieser bezieht sich in seinen instrumentellen und verfahrenstechnischen Einzelheiten auf die Sanierungs- und Abwicklungsrichtlinie (RRD), ist aber wiederum im Hinblick auf eine mit weitreichenden Eingriffsrechten ausgestattete Europäische Abwicklungsbehörde umstritten. Dagegen liegen konkrete Pläne für eine Europäische Einlagensicherung bisher nicht vor. Hauptgrund für das nur zögerliche Vorankommen bei diesen letztgenannten Themen ist die insbesondere in den nordeuropäischen Ländern weit verbreitete Furcht vor einer stillen Sozialisierung national begründeter Schadensfälle. ${ }^{3}$ Auch aus diesem Grunde soll zur Vorbereitung des europäischen Aufsichtssystems (SSM) eine Überprüfung der Werthaltigkeit aller Bankenaktiva durchgeführt werden. Ein in den vergangenen Jahren aufgelaufener und bisher nicht realisierter Wertverlust soll sodann z.B. durch Rekapitalisierungen ausgeglichen werden - eine Voraussetzung für den Start der Bankenunion. Unter

\footnotetext{
${ }^{1}$ Dieser Beitrag greift eine Argumentationslinie auf, die der Autor im April 2013 auf einem Workshop des European University Institute in Florenz vorgestellt hat, vgl. hierzu den im Tagungsband abgedruckten Beitrag „Banking Union in the Eurozone? A Panel contribution“, in: Political, Fiscal and Banking Union in the Eurozone?, hrsg. von F. Allen, E. Carletti und J. Gray, Wharton FIC Press, S. 29-40.

${ }^{2}$ So gibt es insbesondere sehr unterschiedliche Einschätzungen bezüglich der Verankerung der Aufsicht bei der Europäischen Zentralbank und der Abwicklung bei der Europäischen Kommission und ihrer jeweiligen Verankerung in den Europäischen Verträgen.

${ }^{3}$ Eine entsprechende Anzeigenkampagne des Deutschen Sparkassenverbandes und des Genossenschaftsverbandes am 12.09.2012

[http://www.dsgv.de/de/presse/pressemitteilungen/120912_PE_DSGV-BVR_EU-Einlagensicherung.html] hat die hohe Empfindlichkeit deutlich gemacht.
} 
dem Stichwort Asset Quality Review wird bereits jetzt über die Spielregeln einer Aufspaltung alter und neuer Verluste geredet und verhandelt.

Neben der gerade in Deutschland ausgeprägten Sorge um eine europaweite Haftungsvergemeinschaftung gibt es aber auch wettbewerbsbezogene Bedenken bezüglich einer Übertragung der Entscheidungsbefugnis und der Abwicklungskompetenz von der nationalen auf eine europäische (oder transnationale) Ebene. So sehen Verbandsvertreter in Deutschland eine Gefahr für die so genannte Drei-Säulen-Struktur des Bankwesens, weil in der Vergangenheit gewachsene nationale Besonderheiten nicht mehr in dem Maße unangetastet bleiben könnten, wie es im Falle einer primär national orientierten Aufsicht geschehen dürfte ${ }^{4}$.

Beide Sorgen zusammengenommen - Altlasten und nationale Besonderheiten - lassen vermuten, dass es noch eines erheblichen politischen Kraftaktes bedarf, um neben der Abwicklungsproblematik auch die Frage einer europäischen Einlagensicherung im Sinne der gedachten Bankenunion zu einem positiven Ergebnis zu führen. Für die Frage der Haftungsbegrenzung bedarf es einer überzeugenden institutionellen Lösung, für die aber bisher nur vereinzelt Vorschläge vorliegen. ${ }^{5}$

Da das Projekt einer Bankenunion ohne die Unterstützung der wichtigsten Beteiligten, allen voran Deutschland, kaum voranschreiten wird, ist die Tragfähigkeit des Projektes weiterhin in Frage gestellt. Denn tragfähig ist es nur dann, wenn alle vier oben genannten institutionellen Elemente SSM, RRD, SRA und DGS - gleichzeitig in Kraft treten. Diese vier ergänzen sich und bedingen sich in ihrer Wirksamkeit gegenseitig. Werden einzelne Teile nicht umgesetzt, dürfte sich die von einer Bankenunion erhoffte Wiederherstellung marktbezogener Funktionsregeln im Bankensektor nicht entfalten. So wird es insbesondere ohne einen angemessen Abwicklungsmechanismus einschließlich der benötigten Finanzierungsreserve oder-zusage kaum gelingen, die Too-big-to-fail-Problematik zu lösen und staatliche Bankenrettung (und daher auch staatliche Bailouts) wieder zu dem zu machen, was sie immer sein sollten: eine radikale Ausnahmemaßnahme, kein Dauerzustand.

Auf den folgenden Seiten wird eine Kriseninterpretation - in der Literatur heute oftmals als Crisis narrative bezeichnet - vorgetragen, und die wichtigsten Vorschläge des Liikanen-Berichts erläutert (Abschnitt 2). Diese umfassen die Schaffung eines Trennbankensystems für große Institute sowie die Verpflichtung aller Banken zur Emission von Nachrangkapital, das von Nichtbanken gehalten wird. Mögliche Folgerungen für Banken, Investoren und Aufsicht werden in Abschnitt 3 diskutiert. Dabei geht es um angepasste Geschäftsmodelle der Banken, um die Reaktion der Investmentindustrie auf stärkere Haftungsregeln für Privatinvestoren, und um erweiterte Aufgabenstellungen für die Bankenaufsicht. Ein Ausblick auf verbleibende offene Fragen beschließt den Beitrag in Abschnitt 4.

\footnotetext{
${ }^{4}$ Privatbanken, zwei genossenschaftliche Bankengruppen und die öffentlich-rechtlichen Sparkassen und Landesbanken machen etwa 35\%, 20\% bzw. 45\% des Bankensektors -gemessen an den Einlagen und den Kundenforderungen- aus.

${ }^{5}$ Vgl. zu einer dreistufigen europäischen (Rück-) Versicherung mit bergentem Moral Hazard Krahnen (2012) .
} 


\section{Liikanen-Bericht: Deutung der Krise und wichtigste Reformvorschläge}

\section{2.a Die Krisenerklärung}

Die Vorschläge im Liikanen-Bericht sind vor dem Hintergrund der Schilderung der Krise im ersten Teil des Berichts zu verstehen. Dort wird u.a. das Wachstum des Bankensektors in der Europäischen Union (EU-27) seit dem Jahr 2000 anhand von Bilanzdaten beschrieben. In Relation zum Bruttoinlandsprodukt ist der Bankensektor (im Sinne der addierten Bilanzvolumina aller EU-27 Banken) deutlich größer als in den USA. Die entsprechenden Werte für das Jahr 2010 betragen 350\% für Europa und 80\% für die USA. Diese unterschiedlichen Zahlenwerte spiegeln auch die Grösse des Marktes für Unternehmensanleihen in den USA, denen ein relativ kleiner Markt für Unternehmensanleihen (aber ein großer Markt für Bankanleihen) in Europa gegenübersteht. Auch wenn man bei genauerem Hinsehen die Vergleichbarkeit der absoluten Zahlen - etwa wegen unterschiedlicher Rechnungslegungsgrundsätze für Derivatepositionen - durchaus in Frage stellen kann, bleibt die grundlegende Einsicht, dass die Funktionsweise des Bankensektors in Europa eine überragende Rolle für die Spar- und Investitionsprozesse der Volkswirtschaften spielt - und daher die Wiederherstellung eines funktionsfähigen Bankenmarktes in Europa nicht nur einen höheren Stellenwert einnimmt, als etwa in den USA, sondern möglicherweise auch schwerer zu erreichen ist.

Die im Liikanen-Bericht vorgetragene Analyse der Finanzkrise seit 2007 diagnostiziert eine wesentliche und anhaltende Störung der Funktionsweise des Bankenmarktes in Europa. Grundlegend für diese Störung, die sich in niedrigen Fremdfinanzierungskosten und hohen Verschuldungsgraden für Banken äußert, ist der (Dauer-) Zustand eines akuten systemischen Risikos, der in vollem Umfang erstmals nach dem Zusammenbruch der Lehman-Krise erkennbar war. Unter systemischem Risiko ist die gleichzeitige Existenzgefährdung einer Mehrzahl von Finanzinstituten zu verstehen, verbunden mit einer erheblichen Auswirkung auf das Funktionieren der sogenannten Realwirtschaft.

Fast unbemerkt ${ }^{6}$ hatte sich das Risiko systemischer Bankkrisen in den Jahren vor dem Ausbruch der Krise aufgebaut. Diese in der Vergangenheit nicht erlebte Qualität des systemischen Risikos im Finanzsystem ist auf die erheblich stärkere Verflechtung der Finanzinstitute untereinander im Vergleich zu früheren Jahrzehnten zurückzuführen. Durch die in den letzten 25 Jahren rasant gewachsenen Derivatemärkte, die zunehmende Bedeutung besicherter und unbesicherter Ausleihungen am Interbankenmarkt und die erhöhte Abhängigkeit von Refinanzierungsmitteln, die kurzfristig am Kapitalmarkt aufgenommen werden, hat die wechselseitige Abhängigkeit der Institute untereinander erheblich zugenommen. Indirekte Beziehungen, wie die Korrelation von nach dem Marking-to-Market-Prinzip bewerteten Vermögenswerten in den Bilanzen der Banken und die Bedeutung liquiditätssensitiver Marktpreise bei einem Notverkauf ebendieser Wertpapiere, verstärken die gegenseitigen Abhängigkeiten. Direkte und indirekte Abhängigkeiten zwischen Banken schüren wiederum das Risiko, dass Schwierigkeiten einzelner Institute oder dass Preiseinbrüche auf einzelnen Produktmärkten in einem Ansteckungsprozess auf andere Märkte und andere Institute übertragen werden. In einer Art Dominoeffekt werden dann größere Teile des Bankensystems betroffen. Damit wächst die Gefahr, dass grundlegende Finanzdienstleistungen - wie insbesondere Zahlungsverkehr und Kreditversorgung - für die Realwirtschaft nicht mehr flächendeckend zur Verfügung stehen und hier hohe volkswirtschaftliche Kosten entstehen.

\footnotetext{
${ }^{6}$ Aber siehe u.a. die frühen Warnungen von Seiten der Bank für Internatioinalen Zahlungsausgleich), unter der Autorschaft von Claudio Borio und William White (Borio/White 2003), ebenso auch Rajan (2005), in einem vielbeachteten kritischen Beitrag auf der Notenbankkonferenz in Jackson Hole.
} 
Wird ein derartiger Zusammenbruch erwartet, spricht man von einem systemischen Risiko. Tritt dieser Fall ein, sind nahezu immer staatliche Maßnahmen erforderlich, um die dann zu erwartenden erheblichen Kosten für die Realwirtschaft abzuwenden. Diese im Krisenfall unvermeidbare (,zwanghafte'), staatliche Rettung einzelner Institute oder ganzer Institutsgruppen hat unter den gegenwärtigen Umständen eine erhebliche Rückwirkung auf das Verhalten der Akteure am Markt und auf die zu beobachtenden Marktpreise.

Es gilt nämlich grundsätzlich, dass Finanzmarktakteure - Investoren, Kreditnehmer, Gläubiger vernünftigerweise das erzwungene Rettungsverhalten des Staates (d.h. des Steuerzahlers) voraussehen werden und es in die Bewertung von Finanztiteln - und generell in ihr Verhalten einpreisen. Nach den Erfahrungen der Jahre 2007-2012 waren es in erster Linie die Gläubiger, also die Fremdkapitalgeber einschließlich Hybridkapitalgeber, die im Krisenfall mit einer Rettung durch Mittel des Steuerzahlers rechnen durften. ${ }^{7}$ Dagegen mussten Eigenkapitalgeber damit rechnen, dass sie zumindest teilweise, häufig sogar vollständig ihre Kapitalpositionen verlieren.

Diese Erfahrung hat - ganz im Sinne rationaler Erwartungen - zu einer weitreichenden Anpassung der Marktpreise an diese offenbar unvermeidbaren und daher vorhersehbaren Rettungsaktionen geführt. So haben Schweikhard/Tsesmelidakis in einer viel beachteten Studie unter Einschluss von Daten der USA, UK und der EU bereits 2011 gezeigt, dass die (risikoneutralen) Ausfallrisikoschätzungen auf der Basis einerseits von CDS-Preisen und andererseits von Aktienrenditen, bezogen auf die jeweils gleichen Unternehmen, einem einheitlichen Muster folgen. Demnach weichen die beiden genannten Ausfallrisikoschätzer seit etwa Mitte 2007 systematisch voneinander ab, wobei Aktienmärkte ein signifikant höheres Risiko signalisieren, als die Kreditmärkte. Interessanterweise finden sich diese Diskrepanzen in der Risikoeinschätzung im Wesentlichen nur für Banken aber kaum für Nichtbanken. ${ }^{8}$

Zusammengenommen lassen sich die Beobachtungen von Schweikhart/Tsesmelidakis (2011) als Ausdruck eines effizienten, aber dysfunktionalen Marktes erklären. Der Bankenrefinanzierungsmarkt ist dysfunktional, weil die Marktzinsen nicht das tatsächliche Ausfallrisiko widerspiegeln, aufgrund der erwarteten Rettung des Fremdkapitals bei Eintritt eines systemischen Risikofalls, sowie aufgrund der allgemeinen Erwartung, dass ein solcher Fall eine hohe Eintrittswahrscheinlichkeit besitzt. Stattdessen spiegelt der Zins für Bankenrefinanzierung die viel geringeren, verbleibenden Ausfallrisiken nach Berücksichtigung der staatlichen Rettungsaktion wider. Da das gleiche Argument für Eigenkapital nicht gilt, oder zumindest weitaus weniger gilt, fallen beide Preise und damit die beiden Risikosignale systematisch auseinander. Als Konsequenz können sich entgegengesetzte Risikoinformationen aus den Preissignalen in den beiden Märkten für Eigen- und Fremdkapital ergeben. Während der Eigenkapitalmarkt hohe Ausfallrisiken meldet und entsprechend hohe erwartete Renditen einfordert, erscheinen die Ausfallrisiken durch die Linse des Fremdkapitalmarktes betrachtet als gering - eine Fehleinschätzung, die Ergebnis des Dysfunktionalität des Marktes ist.

\footnotetext{
${ }^{7}$ Vgl. die umfassende Sammlung von Fallstudien zur Bailout-Praxis in Europa während der Finanzkrise in Dübel (2013).

${ }^{8}$ Bei den Ausnahmen spielt General Motors, ein Industriebetrieb mit wesentlicher Bedeutung für die USWirtschaft und mit einer herausragenden Rolle als Referenzwerte im amerikanischen CDS eine besondere Rolle. GM hat im Jahre 2009 im Rahmen des TARP-Programms einen Bailout erlebt.
} 
Trotz dieser Dysfunktionalität ist der Markt aber effizient - zumindest im herkömmlichen finanzierungstheoretischen Sinne, nach dem Effizienz für rationale Erwartungen steht, also für die effiziente Verarbeitung aller verfügbaren Informationen.

Der dysfunktionale, effiziente Markt stellt nun aber die Basisinformation für das Risikomanagement der Banken bereit. So sind die impliziten Risikoinformationen in Preisen für Anleihen und Swaps, wie etwa CDS, Teil der steuerungsrelevanten Daten für die bankinternen Risikomodelle. Es ist daher vorstellbar, dass vor und während der Finanzkrise die Risikomanager der Banken, im guten Glauben an die von ihren Märkten empfangenen Signale, - gewissermaßen ahnungslos - immer höhere Risiken eingegangen sind. ${ }^{9}$

Die Argumentationskette endet damit in einem sich selbst verstärkenden Kreislauf (einem ,vicious circle'), in dem vorhandene systemische Risiken zu Rettungserwartungen, und diese zu indirekten Subventionen an die Eigentümer von Banken führen (über subventionierte Refinanzierungskosten). Diese Argumentation fasst die Krisenanalyse zusammen, die dem Liikanen-Bericht zugrunde liegt. ${ }^{10}$

Vor dem Hintergrund dieser Krisenerklärung versucht der Liikanen-Vorschlag nun - wenig überraschend - den soeben geschilderten Teufelskreis zu durchbrechen. Zentrale Idee hierbei ist es, die Abwicklungsfähigkeit einzelner Institute zu stärken und gleichzeitig die Ansteckungseffekte zwischen Banken zumindest auf der Stufe des nachrangigen Fremdkapitals, zu beseitigen. In diesem Zusammenhang ist an eine wiederholt gemachte Erfahrung der Bankenretter - in Deutschland wie auch in anderen Ländern - zu erinnern, die als Wochenendeffekt bezeichnet werden kann. Diese Erfahrung bezieht sich auf den Ablauf einer Bankenkrise, die typischerweise an einem Freitag mit einem Anruf bei einem Bankenaufseher beginnt, in dem die schwierige Lage eines Instituts mitgeteilt wird. Es verbleiben dann etwas mehr als 48 Stunden, nämlich bis zum Öffnen der Tokioter Börse gegen Mitternacht Sonntag/Montag MEZ (und damit der Möglichkeit internationaler Investoren, ihre Anlagen umzuschichten), um eine von den Märkten verstandene Lösung für die Schieflage der Bank zu finden. Das Institut in seiner restrukturierten Form muss dann - insoweit es auf Marktfinanzierung angewiesen ist und damit einem Run von Seiten der nicht einlagengesicherten Einleger ausgesetzt sein kann - in dieser Zeit wieder von außen erkennbar Solvenzstatus erlangen.

Die ernüchternde Erfahrung mehrerer derartiger Wochenendsanierungen mündete sonntags dann in einer steuergeldfinanzierten Rettung, d.h. in einem Bailout. ${ }^{11}$ Hierbei spielte die schiere Komplexität der Geschäftsvorgänge und das hohe Maß an Durchdringung mit oft kurzfristigen marktbezogenen Transaktionen eine besondere Rolle. Vor dem Hintergrund dieser Erfahrung nimmt die Komplexität des Geschäftsportfolios einzelner Banken einen hohen Stellenwert ein, wenn es um die Abwicklungsfähigkeit eines Instituts im Rahmen einer Wochenendaktion geht.

Die beiden vorgeschlagenen Maßnahmen - Abwicklungsfähigkeit stärken, Ansteckungseffekte schwächen - werden im Folgenden genauer beschrieben. Beide zusammen genommen verfolgen das

\footnotetext{
${ }^{9}$ Die Quintessenz dieser Aussage ist, dass es keiner gierigen (usw.) Banker bedarf, um einen Kreislauf aus immer höherer Risikoübernahme gefolgt von staatlichen Rettungsaktionen erklären zu können - es bedarf lediglich einer Externalität (des systemischen Risikos) und rationaler Erwartungen der Akteure.

${ }^{10}$ Es sei hier nur der Vollständigkeit halber angemerkt, dass diese Analyse sich grundlegend unterscheidet von den Krisenanalysen, welche die vor dem Liikanen-Bericht entstandenen Empfehlungen für die USA (VolckerRegel als Teil des Dodd-Frank Act, 2010) und Grossbritannien (Vickers-Report, 2011) begründen.

${ }^{11}$ Diese Aussage lässt sich an den Fallbeschreibungen zur IKB , zur Sachen LB, zur HypoReal Estate überprüfen. Vgl. Spiegel-Online, 7.7.2009, "HRE-Rettung: Top-Banker fürchteten den Tod ihrer Institute", http://www.spiegel.de/wirtschaft/hre-rettung-top-banker-fuerchteten-den-tod-ihrer-institute-a-634716.html
} 
gleiche Ziel: Umfassende Ersetzung der Haftung des Steuerzahlers durch eine Haftung der Bankgläubiger. Falls es gelingt, die Abwicklung von Banken unter Beteiligung der Gläubiger glaubwürdig zu machen, werden die Marktpreise für Bankenfremdkapital wieder die tatsächlichen Risikokosten widerspiegeln, so dass Banken auch weniger riskante Strategien (d.h. Geschäftsmodelle) wählen werden. Als Konsequenz insbesondere einer verminderten Ansteckungsintensität wird schließlich auch das systemische Risiko wieder auf das nicht vermeidbare Mindestniveau sinken können, die jedem funktionalen Bankensystem innewohnt. ${ }^{12}$ Die beiden zentralen Strukturempfehlungen des Liikanen-Berichts werden im Folgenden dargestellt und weitergehend diskutiert. ${ }^{13}$

Die Leitidee der beiden Vorschläge folgt der Logik der vorgestellten Ursachenanalyse der Krise. Die Wiederherstellung privater Haftung im Bankensektor ist die Voraussetzung einer Wiederbelebung der Marktdisziplin - hier vorerst allein auf den Preismechanismus bezogen, später kann das Argument auch auf die Interessenvertretung der Bankgläubiger (also ihrer Einleger) gegenüber dem Bankmanagement und den Eignern angewendet werden. Die beiden Vorschläge sind daher als komplementär zu den vier Bausteinen der Bankenunion zu verstehen. ${ }^{14}$

\section{2.b Trennung der Handelsaktivitäten vom Universalbankgeschäft: Abwicklung erleichtern}

Den größten Raum im Liikanen-Bericht und in den diesbezüglichen Presseberichten nimmt der Vorschlag eines Trennbankensystems mit großzügigen De-minimis Regeln ein, d.h. mit einer Anwendungsbegrenzung auf große Institute. Es bleibt weiter unten zu diskutieren, ob den beiden Vorschlägen mit dieser impliziten Wertung Gerechtigkeit widerfährt. Ungeachtet der eigenen Einschätzung zu dieser Wertungsfrage folgt die Darstellung der Reihenfolge in dem Bericht.

Der erste Vorschlag schlägt eine Zerlegung großer und komplexer Institute in zwei Einheiten vor. Hintergrund ist die in den vergangenen 25 Jahren gewachsene Verzahnung des klassischen, informationsgetriebenen Bankgeschäfts (relationship/commercial banking) und des modernen, Kapitalmarkt-orientierten Handelsgeschäfts (trading/investment banking). Die - letztlich unbewiesene - These hinter diesem Vorschlag ist, dass zumindest ein erheblicher Teil der Komplexität des Bankgeschäfts, die eine Zerlegung und Abwicklung an einem Krisenwochenende unmöglich macht, aus dem Zusammenwirken von Bank- und Handelsgeschäften herrührt. ${ }^{15}$

Vor diesem Hintergrund schlägt der Liikanen-Bericht eine Abspaltung der Handels- von den Geschäftsbankaktivitäten großer Finanzinstitute in zwei rechtlich eigenständige Einheiten vor (,Handelsbank' und ,Geschäftsbank'), die aber unter einem gemeinsamen Holdingdach geführt

\footnotetext{
${ }^{12}$ Zu den Ursachen und den Grenzen der Vermeidbarkeit des systemischen Risikos vgl. auch Bluhm/Krahnen (2011)

${ }^{13}$ Daneben gibt es diverse andere Empfehlungen, unter anderem größere Kapitalpuffer für Handelsaktivitäten und eine Stärkung der Corporate Governance.

${ }^{14}$ Zum Zeitpunkt der Abfassung des Liikanen-Berichts war nicht absehbar, inwieweit der ,große Wurf' einer Bankenunion gelingen kann; die HLEG (Liikanen Kommission) hat daher das Gelingen dieses politischen Großprojektes mit den oben genannten vier Bausteinen (RRD, SSM, SRM, DGS) unterstellt und sich gewissermaßen auf die beiden Schlusssteine konzentriert: Abwicklung erleichtern, Ansteckung vermindern. ${ }^{15}$ Die Liikanen-Kommission hatte während ihrer Arbeit zahlreiche europäische Institute im Hinblick auf ihre Geschäftsmodelle befragt, vgl. European Commission (2012)- Liikanen Report. Auch nach dieser kleinen Anhörung bleiben ernsthafte, empirische Forschungsarbeiten zu diesem Thema eine wichtige Aufgabe für Aufsicht und Wissenschaft.
} 
werden können. ${ }^{16}$ Kapitalisierung und Refinanzierung beider Einheiten müssten jedoch separat erfolgen; eine Garantie darf es nur von Seiten des Handelshauses gegenüber der Geschäftsbank, aber nicht umgekehrt geben. Auf diese Weise soll erreicht werden, dass die implizite Staatsgarantie, die für eine Geschäftsbank schon aufgrund ihrer Depositen besteht, ${ }^{17}$ nicht ohne Weiteres auf die Handelsbank übertragen werden kann. Eine Quersubventionierung des Handelsbereichs durch den Geschäftsbankenbereich mit der entsprechenden Verzerrung der Investitions- und Risikoallokation, soll damit verhindert werden.

Handels- und Geschäftsbanken sollen ihre jeweils eigene Refinanzierung gestalten, und dabei von Seiten des Marktes mit den jeweils eigenen Risikokosten konfrontiert werden. Unter der Annahme, dass in der Vergangenheit eine Quersubventionierung tatsächlich stattgefunden hat, ist als Folge einer Trennung der beiden Geschäftsbereiche zu erwarten, dass die Refinanzierungskosten der Handelsbank steigen und jene der Geschäftsbank sinken. Dieser Preiseffekt würde der Tendenz nach zu einer Reduktion von Handelsaktivitäten am Gesamtmarkt führen. ${ }^{18}$ Allerdings ist es kein Ziel dieser Maßnahme, Handelsgeschäft per se einzudämmen, oder deren volkswirtschaftlichen Nutzen in Frage zu stellen - hierzu gibt es auch aus der Wissenschaft bisher keine konkreten Hinweise. Es geht im Liikanen-Vorschlag allein um die Vermeidung einer eventuellen Quersubventionierung zwischen Geschäfts- und Handelsbank.

Die vorgeschlagene Trennung sieht im Weiteren einen Schwellenwert (De-minimis-Regel) vor, der sich auf die absolute Größe der Bank (100 Milliarden Euro Handelsvolumen) und das relative Volumen der zu Handelszwecken gehaltenen Vermögenswerte bezieht (15-20\% der Bilanzsumme). Das vielleicht wichtigste Detail des Trennbankenvorschlags im Liikanen-Bericht ist die Absicht, zwischen Eigenhandel und Kundengeschäft bzw. zwischen Eigenhandel und Market Making nicht zu unterscheiden. Der Eigenhandel, so das Argument, sei kaum von Handelsaktivitäten für Kunden zu trennen, da das Market Making in nicht absolut liquiden Märkten im Wesentlichen aus einer Abfolge von Geschäften bestehe, die sich letztlich in den Büchern der Bank wiederfinden würden. ${ }^{19}$

Die Trennung von Market Making und Eigenhandel (bzw. Kunden- und Eigengeschäft) großer Institute ist in der Branche auf erheblichen Widerstand gestoßen. Dort wird argumentiert, dass weder die Handelsaktivitäten im Mittelpunkt der Krise standen noch Banken mit umfangreichen Handelsbüchern stärker als andere Institute von der Krise betroffen waren. Weshalb also sollte das Handelsgeschäft ausgegliedert werden und dadurch die Universalbank einschließlich Handels-

\footnotetext{
${ }^{16}$ Betriebswirtschaftliche Einzelheiten einer derartigen Zerlegung werden in dem Bericht nicht angesprochen, weil dies außerhalb der zeitlichen und fachlichen Möglichkeiten einer High-Level Expert Group liegt.

${ }^{17}$ Eine Staatsgarantie kann zwar rechtlich ausgeschlossen werden - allerdings ist zu bezweifeln, dass sich die Handelnden (Zentralbank, Aufsicht, Finanzministerium) in einem akuten Fall systemischen Risikos an eine solche Vorgabe halten kann. Dies erklärt die Verwendung des Begriffs „glaubwürdig“ in dem letzten Satz im Text.

${ }^{18}$ Allerdings sind Vorhersagen über die Auswirkung einer solchen Regeländerung, wie dem hier beschriebenen Trennbankenmodell, deshalb schwierig, weil es sich nicht um eine partielle Verhaltensänderung handelt, sondern um eine Veränderung des gesamten Systems mit einem neuen allgemeinen Gleichgewicht. Es können sich bspw. neue Preise auf dem Märkten für Handelsprodukte ergeben, die den soeben vermuteten Preiseffekt ganz oder teilweise kompensieren.

${ }^{19}$ In den USA schreibt die Volcker-Regel als Teil des Dodd-Frank-Gesetzes den Banken vor, den Handel auf eigene Rechnung vollständig vom übrigen Geschäft zu trennen, während Market Making und das Kundengeschäft nicht angetastet werden. Die Regulierungsvorgaben in Deutschland und Frankreich folgen diesem amerikanischen Beispiel. Nach den Vorstellungen der Liikanen-Kommission ist eine Abtrennung des Eigenhandels aber schwer durchsetzbar, weil moderne Formen des Market Making kaum zu unterscheiden sind vom Eigenhandel.
} 
aktivitäten als Geschäftsmodell an Attraktivität einbüßen? Ferner ist der Wettbewerb auf einem internationalen Markt ungleich schwieriger, wenn nicht für alle die gleichen Bedingungen herrschen.

Mit einer Trennung der Handelsaktivitäten vom übrigen Universalbankgeschäft will der LiikanenVorschlag vor allem die Chance auf eine Abwicklung und Beteiligung der Gläubiger erhöhen. Internationale Universalbanken weisen inzwischen äußerst komplexe Strukturen auf, und ihre Handelsaktivitäten haben wesentlich zu dieser Entwicklung beigetragen. Jeder Versuch, ein in Schieflage geratenes Institut in einer der berüchtigten Blitzaktionen über das Wochenende zu retten, ist zum Scheitern verurteilt, wenn Handels- und klassische Bankgeschäfte eng miteinander verwoben sind.

In den letzten beiden Jahrzehnten haben sich große internationale Kredithäuser zu Spezialisten für Risikomanagement und Absicherungsgeschäfte (Hedging) entwickelt. Dank des massiven Orderflusses und ihrer soliden Stellung an vielen Derivate- und Wertpapiermärkten sind sie in der Lage, Aufträge ihrer Kunden rasch auszuführen und innen Liquidität anzubieten. Durch intensive Nutzung von Netting-Möglichkeiten und innovativen internen Verrechnungsmethoden und Portfoliomodellen kann eine Bank die Zahl und das Volumen von notwendig mit Dritten abzuschließenden Sicherungsgeschäften deutlich verringern. Die damit eingesparten Spreads und Risikoaufschläge kommen dem Ertrag der Bank zugute. Eine derart integrierte Geschäfts- und Handelsbank mit ihren komplexen internen Risiko-Portfolios erschwert aber andererseits eine rasche Sanierung.

Durch die Trennung des Handels- vom Bankgeschäft würden dagegen zwei separate Institute entstehen, ein Handelsinstitut (oder Wertpapierhaus, auch Broker-Dealer genannt) und eine verbleibende (Universal-) Bank. Beide Institute verfügen über ihr separates Eigenkapitalkonto, das von einer gemeinsamen Holding bereitgestellt werden könnte. Während sich die Universalbank weiterhin durch Einlagen, Anleihen und unbesicherte Kredite refinanziert, würde sich das Handelsinstitut das benötigte Kapital selbst beschaffen, möglicherweise an den Anleihe- oder Interbankenmärkten. Es hätte keinen Zugang zum Einlagenmarkt und käme daher nicht in den Genuss einer impliziten Staatsgarantie.

\section{2.c Nachrangbankanleihen für Nichtbanken: Ansteckung vermindern}

Ist ein Trennbankensystem besser gegen systemische Risiken gefeit als ein integriertes Bankensystem ${ }^{20}$ Die Antwort auf diese Frage fällt nicht ganz leicht: sofern nämlich der Eintritt des Risikofalls (wie z.B. ein Run auf die Einlagen einer oder mehrerer Banken, ausgelöst durch einen plötzlichen Kursverlust von Anleihebeständen in den Bilanzen der Institute) vorgegeben ist, kann man keine klare Prognose über ein Mehr oder Weniger an systemischen Risiko treffen. Das systemische Risiko, sprich: das Risiko eines gleichzeitigen Zusammenbruchs mehrerer Institute, kann in einem Trennbankensystem kleiner oder größer sein, als in einem integrierten System. Dabei spielt

\footnotetext{
${ }^{20}$ Der Begriff ,Universalbank' wird an dieser Stelle vermieden - weil auch in dem Trennbankenmodell gemäß Liikanen-Bericht die nach Abspaltung der Handelsaktivitäten verbleibende Bank als Universalbank bezeichnet wird - und werden sollte. Denn ein solches Institut enthält neben den typischen Geschäftsbankenaktivitäten (Depositen, Kredite) auch das Investmentbanking (IPO, SEO, Syndiziertes Kreditgeschäft, M\&A, Private Equity) nur eben kein Handelsgeschäft. Hierfür sind Fremdaufträge zu vergeben, teilweise an die eigene Holding'schwester', teilweise an andere Broker-Dealer im Markt.
} 
u.a. die Eigenkapitalausstattung der Handelsbanken eine Rolle, ebenso die ungesicherten Verbindlichkeiten gegenüber den Geschäftsbanken.

Sofern allerdings der Eintritt des Risikofalls nicht vorgegeben sondern endogen ist, sprich: sich auch aus dem Verhalten der beteiligten Institute ergibt, kann eine Prognose gewagt werden. Da ein Trennbankensystem der Intention nach zu einer verbesserten Abwicklungsfähigkeit einzelner Institute beiträgt, werden auch die Finanzmärkte die Verminderung der impliziten Staatsgarantie vermerken und deshalb einen Anreiz schaffen, die Bankrisiken und die gegenseitigen Abhängigkeiten zu reduzieren. Beides führt zu vermindertem systemischen Risiko, weil Kettenreaktionen quer durch das Bankensystem weniger wahrscheinlich werden.

Ein einfaches Beispiel kann dies illustrieren. Angenommen, die ungesicherten Verbindlichkeiten einer Bank bestehen (auch) aus Forderungen anderer Banken, so wie es beispielsweise im Jahre 2008 bei der deutschen Industriekreditbank (IKB) vermutet wurde. Der geschilderte ,Wochenendeffekt' äußert sich hier in der (begründeten) Befürchtung, dass eine Abschreibung und Wandlung der Bankenforderungen bei den betroffenen Instituten zu einer eigenen Refinanzierungskrise am Folgetag führt - womit möglicherweise das Startsignal für eine systemische Bankenkrise gegeben würde. Angesichts dieser Gefahrenlage wird die Aufsicht nicht anders können, als den Steuerzahler zu Hilfe zu holen und einen Bailout einzuleiten. Diese Maßnahme stabilisiert zwar das Bankensystem an diesem Wochenende - aber um den Preis erhöhter systemischer Risiken in der Zukunft.

Wenn man sich das Beispiel etwas genauer anschaut, erkennt man, dass das eigentliche Problem aus Sicht der Aufsicht in der Identität der Einleger liegt. Weil diese (auch) Banken sind, kann eine Inanspruchnahme privater Haftung (,Bail-in'), wie sie unsere Rechtsordnung eigentlich als Normallfall vorsieht, nicht stattfinden.Wüsste die Aufsicht mit Bestimmtheit, dass die Gläubiger der ungesicherten Bankeinlagen keine anderen Banken sind, so stünde einem Bail-in eigentlich nichts im Wege.

Hieraus leitet sich die Grundidee des zweiten Strukturvorschlags im Liikanen-Bericht ab: Halterestriktionen für Bail-in Anleihen. Durch sie soll die Identität der Käufer von nachrangigen Bankfremdkapital beschränkt werden. Die geforderte Einschränkung ist einfach: Banken sollen Bail-in Anleihen nicht halten. Die Begründung folgt aus dem vorher Gesagten: Wenn die Aufsicht davon ausgehen kann, dass die Inhaber der Bail-in Anleihen selbst keine Banken sind, dann kann sie den Haftungsfall anordnen, ohne unmittelbare systemische Konsequenzen befürchten zu müssen. Und weil dies so ist, wird ihre Ankündigung eines Bail-in auch glaubwürdig ex-ante. Genau diese Glaubwürdigkeit ist aber in Frage gestellt, wenn in einem Moment der Krise Unwissenheit seitens der Aufsicht über die Halteridentität besteht.

Die folgende Geschichte, entnommen der Financial Times mag diese Überlegung unterfüttern.

Die Zeitung berichtet über den Beteiligungsfonds TPG (Texas Pacific Group). Die Gruppe investierte unter Leitung ihres Gründers David Bonderman 2008 etwa 7 Milliarden Dollar in die bereits angeschlagene Bank Washington Mutual. Wie die Zeitung berichtet, setzte der Fondmanager in seiner Anlageentscheidung auf ein umfassendes Bailout durch die amerikanische Regierung (bzw. FDIC). Seine Überzeugung stützte sich auf die vernichtende Erfahrung eines Bail-in bei Lehman-Brothers, kurze Zeit zuvor. Zu TPG's großer Überraschung verweigerte die FDIC jedoch den Bailout und praktizierte statt dessen ein Bail-in. TPG verlor das gesamtes Investment, \$1 Milliarde eigenes Geld und weitere \$6 Milliarden Kundengelder. Als Erklärung für das Verhalten der FDIC - hier kann der Leser nur spekulieren - liegt es nahe 
zu vermuten, dass die Finanzaufsicht den Bail-in deshalb wagen konnte, weil die Identität der Investoren, d.h. TPG als Private Equity Fonds, bekannt war, so dass eine Rückwirkung auf andere Finanzinstitute als unwahrscheinlich eingeschätzt wurde:

"Sheila Blair, the FDIC's chair at the time, described in her memoirs how shocked she had been by Mr. Bonderman's "combative way" in pressing for access to Fed lending. TPG lost all its money (more than $\$ 1 \mathrm{bn}$ ) as well as that of its investors and co-investors, a group that included major sovereign wealth funds and state pension funds. But Mr. Bonderman's admirers point out that if the government had decided to bail out the bank instead, it would have been a fantastic investment." (Private Equity: Last of the risk-takers?, in: Financial Times, European Edition, 19.8.2013, S. 5).

Dieser zweite Vorschlag im Liikanen-Bericht richtet sich daher auf das Kernproblem bis dato gescheiterter Bai-in Versuche und damit auf das Kernproblem der Dysfunktionalität des Bankenrefinanzierungsmarktes. Er setzt direkt an der Ursache für die Allgegenwart des systemischen Risikos im heutigen Bankensektor an. Der Vorschlag verpflichtet Banken - alle Banken - dazu, eine Mindestmenge an unbesicherten Anleihen mit speziellen Haltebeschränkungen auszugeben. Banken zur Ausgabe unbesicherter Anleihen zu zwingen ist an sich nicht weiter bemerkenswert, da die meisten Finanzinstitute ohnehin über eine Fülle im Umlauf befindlicher nachrangiger oder hybrider Instrumente verfügen. ${ }^{21}$

Um etwaigen Missverständnissen vorzubeugen ist zu betonen, dass auch nach der Schaffung von speziellen Bail-in Anleihen alle Positionen der Passivseite einer Bank grundsätzlich und weiterhin der privaten Haftung unterworfen bleiben, also ,bail-in-able' sind. Die Besonderheit dieser Nachranganleihen besteht daher nicht in der Haftung (bail-in), sondern in der Glaubwürdigkeit der Haftungsansage.

Die wesentliche Neuerung des Liikanen-Vorschlags ist deshalb die explizite Haltebeschränkung. Sie sieht vor, dass die vorgeschriebenen nachrangigen Schuldtitel zu keinem Zeitpunkt von einem Institut innerhalb des Bankensystems und damit einem Institut, das einem systemischen Risiko unterliegt, gehalten werden dürfen. Welche Institute außerhalb des Bankensektors als Halter der nachrangigen Schuldtitel in Frage kommen, wird weiter unten diskutiert. Eine Rückübertragung des Ausfallrisikos solcher Nachranganleihen in das Bankensystem, z.B. durch den Erwerb von Credit Default Swaps (CDS), bei denen Banken als Versicherungsverkäufer auftreten, muss aus Konsistenzgründen deshalb ausgeschlossen sein.

Indem nachrangige Verbindlichkeiten außerhalb des Bankensystems platziert werden, können die über das Eigenkapital des betroffenen Instituts hinausgehenden Verluste von den Anlegern getragen werden, ohne einen direkten, ansteckenden Rückkopplungseffekt. Eine Aufsichtsbehörde, die eine Beteiligung nachrangiger Gläubiger an einer Bankenrettung erwägt, kann dann darauf vertrauen, dass ihre Bail-in-Entscheidung nicht die nächste Systemkrise im Bankensektor auslöst: Dieser Sachverhalt kann und soll sie ermutigen, eine Rettung unter Gläubigerbeteiligung durchzuführen. Die Gläubiger wiederum wissen von Anfang an, d.h. vom Tag der Emission an, dass die Gefahr eines Teiloder Totalverlusts ihres Kapitals eine positive Eintrittswahrscheinlichkeit besitzt, und dass der

\footnotetext{
${ }^{21}$ Zu der Rolle nachrangigem Fremdkapitals zur Anreizsteuerung bei Banken gibt es eine lange Literatur, vgl. etwa Calomiris 2000, Calomirirs/Herring (2010) - das Thema einer Haltebeschränkung bleibt allerdings in der gesamten bisherigen Literatur unbeachtet. Der Grund liegt in der Einzelinstitutsperspektive, die in der Literatur vorherrscht. Dagegen findet sich im Liikanen-Bericht eine Berücksichtigung des marktweiten systemischen Risikos.
} 
Steuerzahler nicht - oder zumindest sehr viel seltener - einspringen wird. Entsprechend wird sich das tatsächliche Ausfallrisiko - Markteffizienz unterstellt - in den Marktpreisen widerspiegeln, was gleichbedeutend ist mit der ursprünglich gewünschten Marktdisziplin.

Laut Liikanen-Bericht kann die strikte Haltebeschränkung auch durch eine Auflage ersetzt werden, die es Banken gestattet, in nachrangige Schuldtitel von Banken zu investieren. Allerdings sind sie dann verpflichtet, diese Titel vollständig mit Eigenkapital zu unterlegen (Risikogewicht 1250\%). Beide Regeln bewirken im Wesentlichen dasselbe: Nachrangige („Bail-in“) Bankverbindlichkeiten werden außerhalb des Bankensystems gehalten. Hierdurch würden die Rückkopplungseffekte von Gläubigerbeteiligungen auf die Stabilität des Finanzsystems auf ein Minimum reduziert. Durch die Halterestriktion wird das betreffende Kapital Bail-in-fähig.

\section{Folgerungen für Banken, Investroren und Aufsicht}

Weite Teile des regulatorischen Reformprojekts der letzten Jahre wurden von der Finanzindustrie begrüßt. Die im Regelwerk Basel III festgeschriebenen Neuerungen in Bezug auf eine Stärkung der Eigenkapitalbasis und die Einführung einer Verschuldungsgrenze sind beispielsweise auf ein überwiegend positives Echo gestoßen. Das Projekt einer Bankenunion, in dessen Mittelpunkt eine gemeinsame Aufsicht und Abwicklung innerhalb der Europäischen Union steht, findet ebenfalls breite Unterstützung. Bei bestimmten, für nationale Institute und deren Geschäftsmodelle wichtigen Themen stößt das Projekt jedoch auf erheblichen Widerstand. Zudem gibt es Bedenken hinsichtlich der künftigen Rolle der nationalen Aufsichtsbehörden. Entsprechend gering ist ganz allgemein die Bereitschaft, die Zuständigkeiten von der nationalen auf die supranationale Ebene zu übertragen. ${ }^{22}$

Nach Vorlage des Liikkanen-Berichts sind zahlreiche Fragen zu Einzelheiten einer möglichen Ausgestaltung der Regulierung aufgekommen. Auf einige dieser Fragen soll im Folgenden ohne Anspruch auf Vollständigkeit eingegangen werden. Dem Aufbau der bisherigen Ausführungen folgend werden zuerst drei häufig aufkommende offene Fragen zum Trennbankenmodell angesprochen: welche Auswirkungen sind von einem Trennbankenmodell auf die Profitabilität einer Bank, den Wettbewerb zwischen Banken, und die Liquidität auf Wertpapiermärkten zu erwarten?

Im Anschluss werden mögliche Auswirkungen der Einführung einer Emissions- und Halteregulierung für nachrangiges Bankfremdkapital (Bail-in Kapital) diskutiert. Hier werden drei Aspekte angesprochen: Wer sollte die nachrangigen Anleihen halten, wenn Banken daran gehindert werden? Wird es einen ausreichend großen Investorenkreis geben? Soll die Halterregelung überwacht werden - und von wem?

\footnotetext{
${ }^{22}$ Bei der gemeinsamen Bankenaufsicht ist bislang nach wie vor unklar, welche Befugnisse die EZB (als Aufsichtsbehörde) über die größten Banken haben wird und welche Entscheidungs- oder Vetorechte bei den nationalen Behörden bleiben. Dieser Punkt ist besonders für Entscheidungen mit fiskalischen Konsequenzen wichtig, wie etwa im Falle einer Bankenabwicklung. Für den einheitlichen Abwicklungsmechanismus wurde bisher noch nicht entschieden, welche Entscheidungskompetenzen übertragen werden sollen.
} 


\section{3.a Folgeüberlegungen zum Trennbanken-Vorschlag}

Insgesamt sind die Vorschläge im Liikanen-Bericht zum Trennbankensystem auf Seiten der Industrie überwiegend auf Ablehnung gestoßen. In Deutschland argumentieren beispielsweise die Verbände aller drei Säulen des Bankwesens, der öffentlich-rechtlichen Sparkassen und Landesbanken, der Privatbanken und der Genossenschaftsbanken, dass eine Abtrennung der Handelsaktivitäten das bewährte Universalbankenmodell gefährdet. Jede der drei genannten Parteien führt für ihre Ablehnung unterschiedliche Argumente ins Feld, was sich aus den unterschiedlichen Organisationsund Geschäftsmodellen erklären lässt.

Eine umfassende Studie über die tatsächlich zu erwartenden Auswirkungen kann an dieser Stelle nicht geleistet werden. Die nachfolgenden Kommentare sind der Methode nach spekulativ, sie können als Auftakt zu einer Diskussion verstanden werden, an deren Ende hoffentlich Hypothesen stehen, die mit wissenschaftlicher Methodik getestet werden können.

\section{3.a.i Verminderte Profitabilität?}

Ein häufig gehörtes Argument betrifft die Profitabilität reiner Wertpapierhäuser (sogenannter Broker-Dealer). Nach Ansicht einiger Kommentatoren wird die im Trennungskonzept implizierte eigenständige Kapitalbeschaffung höhere Refinanzierungskosten für Wertpapierhäuser mit sich bringen. Dies würde ggfs. zu Lasten der Profitabilität des Market Making gehen. In der Folge würde weniger Liquidität an den Märkten bereitgestellt, an denen Market Maker dominieren. Eine in den USA im Auftrag der Handelskammer durchgeführte Auswirkungsstudie hat die erwarteten Konsequenzen einer Umsetzung der Volcker-Regel geschätzt. Sie kommt zu dem Schluss, dass sich die Umsetzung negativ auf die Marktliquidität auswirkt (vgl. Thakor 2012).

Allerdings ist die Studie nicht gänzlich überzeugend, da die Auswirkungen eines Kostenanstiegs für ein einzelnes Finanzinstitut untersucht wird, während die Auswirkung einer allgemeinen Gesetzesänderung auf das allgemeine Gleichgewicht außer Acht gelassen wurden. Mit anderen Worten: Eine für alle Institute am Markt geltende Trennung der Handelsaktivitäten (und vor allem des Market Making) würde abgesehen von den Refinanzierungskosten der Wertpapierfirmen wohl auch andere Preisänderungen mit sich bringen. Insbesondere wäre zu erwarten, dass sich die Dienstleistungen des Market-Making nach einer derartigen Neuregelung ebenfalls verteuern würden und der von Intermediären verdiente Spread dadurch steigen würde. Bei den Auswirkungen auf die Profitabilität der Broker-Dealer sind deshalb wohl drei Anpassungen zu berücksichtigen: die Refinanzierungskosten (nach oben), der Preis für Market Making-Dienste (nach oben) und das Transaktionsvolumen (voraussichtlich nach unten). Der daraus resultierende Spread und somit die Profitabilität der Broker-Dealer lässt sich nur schwer vorhersagen. Denkbar wäre sogar, dass ein neues Marktumfeld, gekennzeichnet durch die gesetzlich vorgeschriebene Trennung des Market Making und Eigenhandels vom Universalbankgeschäft, die Zahl der Institute, die solche Dienstleistungen anbieten, verringert und die Profitabilität aus dem Handel für die verbleibenden Institute erhöht. $^{23}$

\section{3.a.ii Aufsichtsarbitrage?}

Ein weiteres Argument, das gegen die Trennungsvorschrift vorgebracht wird, stellt den im Bericht vorgeschlagenen Schwellenwert (De-minimis-Regel) in Frage. Demnach müssen nur Banken mit umfangreichen Handelsbüchern ihre Handelsaktivitäten auf eine getrennte Einheit übertragen. Der

\footnotetext{
${ }^{23}$ Dies wäre dann der Fall, wenn sich positive Skaleneffekte für Wertpapierfirmen ergeben.
} 
Bericht schlägt vor, bei einem absoluten Handelsbuchvolumen von 100 Milliarden Euro (oder wenn das Handelsbuch 15-25\% der Bilanzsumme übersteigt) die Grenze zu ziehen.

Würde eine derart großzügige De-minimis-Regel mittelgroße Banken nicht geradezu einladen, ihre Handelsgeschäfte bis auf ein Niveau knapp unterhalb des Schwellenwerts auszuweiten? Und würde eine derartige Marktentwicklung größere Institute nicht eines erheblichen Geschäftsvolumens berauben und ihr separates Broker-Dealer-Geschäft ineffektiv bzw. unprofitabel machen?

Eine zu beobachtende Konzentration von Handelsaktivitäten bei immer weniger großen Marktteilnehmern lässt das oben beschriebene Szenario als wenig wahrscheinlich erscheinen, sofern in der Wertpapierbranche echte Größenvorteile vorhanden sind. Ohne diese Größenvorteile würde vermutlich schon heute eine dezentrale Handelsarchitektur vorherrschen und es würde keine (weiteren) negativen Auswirkungen auf die Abwicklungsfähigkeit von Banken geben, da die Komplexität von Universalbanken mit relativ geringem Handelsgeschäft als beherrschbar gilt.

Würden indes künftig einige große Broker-Dealer den Markt beherrschen, können diese Institute aufgrund ihrer starken Verflechtung mit zahlreichen Nicht-Broker-Dealer-Bankinstituten in ganz Europa ein signifikantes systemisches Risiko darstellen. Daher empfiehlt der Liikanen-Bericht, die Eigenkapitalanforderungen für Handelsinstitute weiter zu verschärfen.

\section{3.a.iii Weniger Liquidität?}

Die Marktliquidität wird heute vor allem durch große internationale Universalbanken mit umfangreichen Handelsbüchern (held to maturity oder available for sale) sichergestellt. Inwieweit aber hängt ihr Geschäftsmodell vom uneingeschränkten Zugang ihrer Kunden zum Orderfluss ab? Oder anders formuliert: Ist es möglich, dass eine solche Bank ihren Kunden auch dann das gewohnte Dienstleistungsspektrum anbieten kann, wenn das Handelsgeschäft in ein separates Wertpapierhaus überführt wurde?

Es scheint zumindest denkbar, dass ein neu geschaffenes, separates Wertpapierhaus die Universalbank weiterhin in den Bereichen Finanzstrategie und Risikomanagement berät und die Ausführung der Transaktionen externen Broker-Dealern auf dem Markt überlässt. Die Profitabilität von Wertpapierdienstleistungen würde dadurch zwar möglicherweise sinken, jedoch ohne dass dies zwingend einen Rückgang der Profitabilität der Institute bedeuten müsste. Prinzipiell wäre auch möglich, dass die Gewinne auf aktuellem Niveau verharren, beispielsweise wenn die Ausübungspreise steigen oder für die beim „alten“ Wertpapierhaus verbleibenden Beratungsleistungen ein angemessener Preis gezahlt wird. Diese ausgleichenden Preiseffekte sind in ihrer Gesamtwirkung schwer abschätzbar, da durchaus neue Akteure auf die Wertpapiermärkte drängen und so ein gewisser Druck auf die Profitabilität entstehen könnte.

\section{3.b Folgeüberlegungen zur Schaffung von Bail-in Kapital}

Wer sollte die nachrangigen Anleihen halten, wenn andere Banken daran gehindert werden? Wird es einen ausreichend großen Investorenkreis geben? Soll die Halterregelung überwacht werden - und von wem?

Das Fehlen eines tatsächlichen Risikotransfers außerhalb des Bankensystems hat die Finanzkrise erst ins Rollen gebracht wie die Allokation von ABS-Tranchen eindeutig belegt (siehe z.B. Franke/Krahnen 
2009). Statt das Risiko auf die Investoren zu übertragen, wurde es zumindest teilweise zwischen Finanzinstituten hin und her verschoben. Die Folgen waren eine zunehmende Verflechtung und Ansteckung sowie ein Anstieg des systemischen Risikos.

Der Liikanen-Vorschlag sieht vor, Banken das Halten von Nachranganleihen anderer Banken zu untersagen und sie gleichzeitig dazu zu zwingen, Nachrangpapiere auszugeben - was sicherlich zu einem erheblichen Risikotransfer beitragen wird. Und darin besteht tatsächlich das Hauptziel der (eigentlich unzutreffend bezeichneten) „Bail-in-Instrumente“. Die Schaffung einer Ebene mit Nachranganleihen von Banken, von denen keine Ansteckung innerhalb des Bankensystems ausgehen kann, stellt naturgemäß eine Art nichtsystemisches Risiko dar. Der damit einhergehende Risikotransfer ist daher glaubwürdig, da eine Rettung der Gläubiger durch den Staat nicht zu erwarten ist.

Weitere Anforderungen an glaubwürdiges Bail-in zielen auf die Sicherstellung eines dauerhaften Kosteneffektes für Banken. Hierfür ist sicherzustellen, dass die emittierten Bail-in Anleihen endliche Laufzeiten haben (keine consols sind) und dass sie zeitlich versetzt fällig werden (staggered maturities). Ersteres soll verhindern, dass bei Erreichen eines ausreichenden Bail-in Volumens weitere Emissionen unnötig werden und damit die vereinbarten Kuponzahlungen unabhängig vom tatsächlichen Risiko der Bank werden. Letzteres stellt sicher, dass es bei hohem Bail-in Kapitalvolumen zu keinem sprunghaften Refinanzierungskosteneffekt kommt, der die Solvenz der Bank gefährden und einen Run auslösen könnte. ${ }^{24}$

\section{3.b.i Wer ist Risikoträger für Bail-in Fremdkapital?}

An die zukünftigen Halter von nicht-systemischen nachrangigem Bankfremdkapital sind Anforderungen zu stellen: sie dürfen sich nicht ihrerseits im Bankensektor rückabsichern und sie sollten nicht selbst in Refinanzierungsschwierigkeiten geraten, wenn sie Bail-in bedingte Verluste auf ihre Vermögensanlagen erleiden. Als geeignete Investoren erscheinen daher diversifizierte Kapitalanlagegesellschaften mit langen Vertragslaufzeiten auf der Passivseite, restriktiven Kündigungsrechten und variablen Auszahlungsversprechen. Auf dieses Profil passen insbesondere (deutsche) Lebensversicherungsgesellschaften, aber auch Sovereign Wealth Funds, Pensionsfonds, vermögende Einzelpersonen und spezialisierte Hedge Fonds. In allen Fällen beruht die Attraktivität einer Anlage auf dem erwarteten hohen Kupon.

Soweit der Markt informationseffizient ist, und das angekündigte Bail-in glaubwürdig, wird der am Kapitalmarkt vom Emittenten zu zahlende Kupon eine Höhe erreichen, die dem erwarteten Verlust aus dem Besitz einer Bail-in Anleihe vollständig abdeckt, und darüberhinaus eine Risikoprämie beinhaltet. Für eine Versicherungsgesellschaft liefert der hohe Kupon also erst nach langjähriger Rückstellungsbildung einen ausschüttungsfähigen Gewinn - die zuvor auflaufenden hohen Erlöse sind als Rückstellungen für drohende Verluste aus schwebenden Geschäften zu verbuchen. Sofern Investoren, insbesondere Lebensversicherungen diese Tatsache beachten - und nicht unzulässiger Weise Ergebnisbeteiligungen an Kunden ausschütten, wird der Investorenkreis über einen robusten Schockabsorptionsmechanismus verfügen und damit das System stabilisieren. In diesem Fall kann aus der Sicht des Finanzsystems von einem erfolgreichen, weil rückwirkungsfreien Transfer von Bankrisiken gesprochen werden.

\footnotetext{
${ }^{24}$ Für diesen Hinweis danke ich Frank Heinemann.
} 


\section{3.b.ii Entsteht ein ausreichend großer Markt?}

Ein vielfach in den Diskussionen vorgetragenes Argument bezweifelt die Existenz eines ausreichend großen Marktes, um die im Liikanen-Bericht geforderten Nachranganleihen aufzunehmen. Um dieses Argument zu bewerten, ist die vermutete Größe eines Marktes für Banknachranganleihen abzuschätzen. Überschlägige Berechnungen auf der Basis einer Vorgabe von 5\% der Bilanzsumme und Daten des Jahres 2012 verweisen auf ein Emissionsvolumen in der Größenordnung von 2 Billionen Euro. Dieser Betrag ist etwa 5-6 mal so hoch wie der europäische Junk-Bond Markt des gleichen Jahres. ${ }^{25}$ Vor dem Hintergrund dieser Zahlen ist das Vorhaben, einen 5\%-Anteil an den Bilanzen europäischer Banken in Form von Anleihen mit eine Halterestriktion zu emittieren, ein ehrgeiziges Ziel.

Um dieses Ziel zu erreichen, ist auf vermutete Anforderungen der Investoren schon bei der Konzeption der Instrumente zu achten. Eine neue Assetklasse, Bail-in bonds' sollte von daher standardisierte Anleihekonditionen aufweisen, um die Entstehung eines liquiden Sekundärmarktes zu fördern. Weitere Anforderungen betreffen den Bail-in Prozess selbst, die Auslösung des Haftungsfalles und die Form der Risikoteilung.

So ist die Auslösung des Haftungsfalles so weit als möglich vorab zu definieren, um eine Bewertung durch den Kapitalmarkt zu erleichtern. Gespräche mit Investoren verweisen auf die Rolle sogenannter exogener Trigger. Dies sind vorher festgelegte Auslöser für den Haftungsfall, die möglichst weitgehend diskretionärer Entscheidung durch die Aufsicht entzogen sind. Ein exogener Trigger ist bspw. die Festlegung eines Mindesthaftungskapitals, bei dessen Unterschreitung der Haftungsfall eintritt. Andere denkbare exogene Trigger sind Marktpreise, wie etwa der Aktienkurs oder der Preis für Kreditversicherungen (CDS) der jeweiligen Bank. In beiden Fällen ergeben sich weiterführende Fragen: Inwieweit ist ein exogener Trigger tatsächlich ,exogen', d.h. inwieweit ist er anfällig für eine gezielte Beeinflussung durch das betreffende Kreditinstitut, oder durch Dritte - und inwieweit wird dadurch die Rolle privater Haftungsträger zur Vermeidung systemischer Ansteckung betroffen? Ebenso stellt sich die Frage, inwieweit die Vorgabe eines exogenen Triggers zu einer fehlerhaften (type-Il error) Auslösung des Haftungsfalles führen kann? Schließlich: Wie sieht eine optimale Kombination von exogenem und diskretionärem Trigger aus, welche die Interessen der Investoren nach Berechenbarkeit erfüllt.

Eine weitere Anforderung an die Emissionsbedingungen betrifft die durch ein eventuelles späteres Bail-in induzierte Form der Risikoteilung. Die in den letzten Jahren üblicherweise praktizierte Form der Gläubigerhaftung nimmt eine Wertberichtigung in Form einer Abschreibung des Nominalwerts der Forderung vor. Obwohl dies wertmäßig zum jeweiligen Zeitpunkt identisch ist mit einer Wertkorrektur mittels Umwandlung von Fremd- in Eigenkapital, unterscheidet sich die implizite Risikoteilung in beiden Fällen erheblich. Im zweiten Fall, einem Debt Equity Swap (DES), behält die haftende Gläubigerin eine Beteiligung an dem Unternehmen und im Falle einer zukünftigen Erholung partizipiert sie an einer eventuellen zukünftigen Werterholung des Eigenkapitals. Die Teilhabe an einer potentiellen Wertsteigerung ist eine Möglichkeit die Kosten eines Fehlalarms (type-Il error) in Grenzen zu halten.

Die für den Krisenfall zu planende Eigentümerstruktur spricht ebenfalls für eine Umwandlung von Fremd- in Eigenkapital, anstelle einer Abschreibung. Denkbar ist eine Verwässerung in abgestufter Form, beginnend mit dem höchsten Maß an Verwässerung für die Alteigentümer, gefolgt von einer

\footnotetext{
${ }^{25}$ Das aggregierte Bilanzvolumen der EU-27 Banken beträgt etwa 13 Billionen Euro.
} 
geringeren Verwässerung (gegenüber dem ursprünglichen Nominalanspruch) für die Nachranggläubiger. Bei weiterer Wertminderung des Institutsvermögens können sukzessive auch vorrangige (senior) Gläubiger dem Bail-in in gleicher Form (Wandlung und Verwässerung) unterworfen werden. Dadurch gilt die Grundregel privater Haftung grundsätzlich für alle Passivpositionen, einschließlich der Depositen. ${ }^{26}$ Auf diese Weise würden den Instituten die ursprüngliche Eigentümer- und Gläubigerstruktur zumindest der Zusammensetzung nach erhalten bleiben. Dagegen ergeben sich bei schrittweiser Totalabschreibung der Eigentümer- und nachrangigen Gläubigerposition erratische und von der Größe der Wandlungsschritte abhängige - ggfs. temporäre - Eigentümerstrukturen. Dies erschwert aufgrund der notwendigen Aufsichtsratsbesetzungen nicht nur die Unternehmenskontrolle (Governance) in der Krise, es könnte auch die Bewertung dieser Instrumente mit einer zusätzlichen Risikoprämie versehen.

\section{3.b.iii Welche Anforderungen stellen sich an die Aufsicht?}

Die glaubwürdige Ankündigung eines Bail-in, der ggfs. zu transparenten Bedingungen ohne Manipulationsspielraum durchgeführt wird, ist eine Voraussetzung für einen funktionierenden Markt mit Bail-in Instrumenten. Dieser wiederum signalisiert dem Risikomanagement der Bank die Risikowahrnehmung des Marktes und schafft damit die Voraussetzung für eine direkte Wirkung von Marktdisziplin. Um diese Zielsetzung zu erreichen, ist neben den im letzten Abschnitt beschriebenen Gestaltungsmerkmalen der Instrumente zu gewährleisten, dass ein Bail-in jederzeit praktiziert werden kann, ohne dass die ausführende Behörde befürchten muss, durch ihre Handlung einen systemischen Risikoschock für das Bankensystem auszulösen.

Wenn diese letzte Bedingung erfüllt ist, kann von einer Glaubwürdigkeit der Bail-in Ankündigung gesprochen werden. Diese Bedingung sicherzustellen wird zu einer neuen Aufgabenstellung, die zweckmäßigerweise bei der Bankenaufsicht unterzubringen ist. ${ }^{27}$ Die Überwachung der Bail-inFähigkeit ist ein Kernstück des im Likkanen-Bericht skizzierten neuen Ordnungsrahmens für die Europäischen Finanzmärkte. Sie erfordert mehrere Einzelschritte, in deren Verlauf sich neue Anforderungen an die Datenlage der Aufsicht ergeben. Hierzu zählt u.a. die jederzeitige Kenntnis über die Halteridentität von Bail-in Anleihen. Damit verbunden ist die Möglichkeit, den Kreis potentieller Halter einzuengen. Weiterhin ist sicherzustellen, dass kein Transfer der Ausfallrisiken der Anleihen in den Bankensektor etwa mittels Kreditversicherungen (CDS) oder anderer Instrumente erfolgt. Insgesamt sorgt die Aufsicht damit selbst für das notwendige (Selbst-) Vertrauen in eine Auslösung des Triggers ohne Furcht vor unmittelbaren systemischen Konsequenzen im Moment der Schieflage eines einzelnen Bankinstituts.

Ferner muss betont (betont!) werden, dass der im Liikanen-Bericht geprägte Begriff „Bail-inInstrument" nicht etwa bedeutet, dass es sich bei allen anderen Arten von (Bank-) Verbindlichkeiten um Bail-out-Instrumente handelt. Das Gegenteil ist der Fall. Dem Bericht zufolge sind sämtliche Verbindlichkeiten von Banken gemäß ihrer Stellung in der Rangfolge der Schuldinstrumente bail-infähig, können also für eine Rettung herangezogen werden. Nur die unterste Tranche, hier Bail-in Kapital genannt, muss eine Mindestgröße aufweisen (z.B. 5\% der Bilanzsumme) und unterliegt den zuvor beschriebenen Haltebeschränkungen.

\footnotetext{
${ }^{26}$ Bei Depositen greift dann unmittelbar die Einlagenversicherung bis zu einem garantierten Höchstbetrag.

${ }^{27}$ Unter Umständen kann auch die Marktaufsicht mit der Sicherstellung der Bail-in Glaubwürdigkeit mandatiert werden; dies wär im Falle Europas dann eine Aufgabe der ESMA in Zusammenarbeit mit der EZB als Träger einer einheitlichen Aufsicht, sowie ggfs. mit der nationalen Bankenaufsicht.
} 


\section{Ausblick und offene Fragen}

Die Zukunft des europäischen Bankensektors im Jahre 2013 hängt wesentlich von dem Willen der verantwortlichen Politiker in Europa ab, eine Liste von neuen Gesetzen und Ausführungsverordnungen umzusetzen, die in ihrer Gesamtheit ein geschlossenes Gesamtbild ergeben. Es bedarf dabei des Mutes der Politiker, um über den Schatten der nationalen Budgethoheit zu springen und supranationale, europäische Institutionen als Ersatz für nationale Hoheit hinzunehmen. All dies wiederum dient der Wiederherstellung eines Ordnungsrahmens, der einer Marktdisziplin Geltung verschafft. Ansatzpunkte sind hierfür insbesondere die Preise für das nachrangige Fremdkapital für Banken. Der Liikanen-Vorschlag kann demnach zu einer Stabilisierung der Finanzintermediation in Europa und zu einer Reparatur des marktwirtschaftlichen Ordnungsrahmens wesentlich beitragen.

Bei der Umsetzung und Implementierung kommt es allerdings nur am Rande auf die ökonomische Stringenz an. Bedeutender wird dann die politische Mehrheitsfähigkeit. Hier spielt die Reihenfolge mit der die Einzelthemen der Bankenunion angegangen werden und die Qualität der Sicherungen gegen eine vorschnelle Gemeinschaftshaftung eine zentrale Rolle. Es soll daher herausgestellt werden, dass die in diesem Papier diskutierten Vorschläge der Liikanen-Kommission sich besonders eignen, um die Glaubwürdigkeit in der Öffentlichkeit für eine Gültigkeit privater Haftung bei Banken, auch bei großen Banken, wiederherzustellen. Es bleibt abzuwarten, ob eine solche vernünftige, zielgerichtete Institutionenbildung im Kreis der EU-27 oder EU-28 ohne die Katharsis einer erneuten Bankenkrise möglich ist. 


\section{Quellenverzeichnis}

Bluhm, M. und J. Krahnen (2011). Default risk in an interconnected banking system with endogenous assets, CFS Working Paper No. 19,. Center for Financial Studies, Frankfurt.

Borio, C. und W. White (2003). Whither monetary and financial stability? The implicatioins of evolving policy regimes. Proceedings of the Federal Reserve Bank of Kansas City Symposium at Jackson Hole.

Calomiris, C. und C. Kahn (1991). The role of demandable debt in structuring optimal banking arrangements. American Economic Review, Vol. 81, No. 3, S. 497-513.

Calomiris, C. und R. Herring (2011). Why and how to design a contingent capital debt requirement. Working Paper Columbia University und Wharton School, U Penn.

Dübel, A. (2013). The capital structure of banks and the practice of bank restructuring. Case Studies on current bank restructurings in the Eurozone and conclusions for the reform of resolution and restructuring and regulation of funding Instruments. CFS Working Paper, Center for Financial Studies, Frankfurt.

Europäische Kommission (2013). Vorschlag für eine RICHTLINIE DES EUROPÄISCHEN PARLAMENTS UND DES RATES zur Festlegung eines Rahmens für die Sanierung und Abwicklung von Kreditinstituten und Wertpapierfirmen und zur Änderung der Richtlinien 77/91/EWG und 82/891/EG des Rates, der Richtlinien 2001/24/EG, 2002/47/EG, 2004/25/EG, 2005/56/EG, 2007/36/EG und 2011/35/EG sowie der Verordnung (EU) Nr. 1093/2010, COM(2012) 280. Brüssel, Juni.

European Commission (2012). Report by the High Level Expert Group on Structural Reforms of the EU Banking Sector. Brussels, October.

Franke, G. und J. Krahnen (2009). The future of securitization. ,In: Prudent lending restored: Securitization after the mortgage meltdown (Eds. Fuchita Y., Herring J., Litan E.; Brookings Institution), S. 105- 161

Krahnen, J. (2012). Europataugliche Einlagenversicherung: Vorschlag für eine dreistufige Einlagensicherung mit begrenzter europäischer Haftung. Policy Letter No. 16, Policy Platform at the House of Finance, Goethe-Universität Frankfurt.

Rajan, R. (2005). Has financial development made the world riskier? Proceedings of the Federal Reserve Bank of Kansas City Symposium at Jackson Hole.

Schweikhard, F. und Z. Tsesmelidakis (2012). The impact of government interventions on CDS and equity markets, SSRN Working Paper .

Thakor, A (2012). The economic consequences of the Volcker rule. Report by the US Chamber's Center for Capital Market Competitiveness. 